\title{
КОМПЛЕКСНЫЙ МЕТОД ПОЗИЦИОНИРОВАНИЯ МАРКИРОВАННЫМИ АУДИОСИГНАЛАМИ В ПОМЕЩЕНИИ ${ }^{1}$
}

\section{COMPREHENSIVE INDOOR POSITIONING WITH WATERMARKED DIGITAL AUDIO SIGNALS ${ }^{2}$}

\section{Gofman}

Summary. The article develops an complex method of positioning in a room, when as a communication channel, between a mobile point (smart device equipped with a microphone) and reference points (speakers of the room speaker system), an airborne audio channel is used, through which marked audio signals are transmitted. A feature of the method is that it uses a traditional approach based on solving a system of hyperbola equations, as well as an analytical approach based on finding the minimum of the objective function. The article presents the results of field experiments on positioning with marked audio signals in the room. Based on the results of field experiments, a statistical assessment of the positioning accuracy by the developed complex method was carried out. The standard deviation and the average value of the distances between the coordinate of the point of the true location of the microphone and the coordinates of this point, found as a result of applying the proposed complex method to the data obtained from field experiments on the transmission and reception of marked audio signals, are calculated.

Keywords: steganography, audio stegosystem, digital audio signal, audio watermarking, positioning, indoor positioning.

\section{Введение}

$\Pi$ омещение, представляя собой место в пространстве, ограниченное со всех сторон, зачастую позволяет оградить акустическую систему связи от шумов различного происхождения, например, городского или природного происхождения. В помещении можно ожидать меньший уровень постороннего акустического шума, в сравнении с воздушным аудиоканалом вне помещений, когда осуществляется вещание аудиосигналов, переносящих информацию, полезную для позиционирования.
Гофман Максим Викторович

К.т.н., дочент, Петербургский государственный университет путей сообщения Императора

Александра I

maxgof@gmail.com

Аннотация. В статье разрабатывается комплексный метод позиционирования в помещении, когда в качестве в качестве канала связи, между мобильной точкой (оснащенное микрофоном смарт устройство) и опорными точками (динамики акустической системы помещения), используется воздушный аудиоканал, по которому передаются маркированные аудиосигналы. Особенностью разрабатываемого метода является то, что в нем применяется традиционный подход, основанный на решении системы уравнений гипербол, а также аналитический подход, опирающийся на поиск минимума целевой функции. В статье приведены результаты натурных экспериментов по позиционированию маркированными аудиосигналами в помещении. На основании результатов натурных экспериментов выполнена статистическая оценка точности позиционирования разрабатываемым комплексным методом. Вычислены стандартное отклонение и среднее значение расстояний между координатой точки истинного месторасположения микрофона и координатами этой точки, найденными в результате применения предлагаемого комплексного метода к данным, полученным из натурных экспериментов по передаче и приёму маркированных аудиосигналов.

Ключевые слова: стеганография, аудиостегосистема, цифровой аудиосигнал, маркирование аудиосигналов, позиционирование, позиционирование в помещении.

В статьях [1-5] содержится обзор подходов для решения задачи позиционирования в помещении с помощью радиосигналов. Определение местоположения с помощью акустических сигналов может оказаться более точным подходом, чем подходы, использующие радиосигналы, когда протокол синхронизации между передатчиком и приёмником не может быть установлен. Так как из-за относительно низкой скорости звука в сравнении со скоростью света (обычно применяемой для оценки скорости распространения радиосигналов) небольшая ошибка в синхронизации в системах позиционирования, использующих аудиосигналы, приведёт 
к значительно меньшей ошибке определения месторасположения, в сравнении с системами позиционирования, использующими радиосигналы.

Системы позиционирования обычно предполагают наличие некоторого числа опорных узлов, развёрнутых в заданных точках пространства, а также один или несколько мобильных узлов. Эта терминология не является универсальной, и часто зависит от технологии, положенной в основу. Например, в системах отслеживания, использующих сотовые сети связи, термин базовая станция используется для обозначения опорных узлов, тогда как термин мобильный телефон используется для обозначения мобильных узлов.

Помещения зачастую оборудованы акустическими системами. Так, по технике безопасности в помещениях обязательно должны присутствовать системы экстренного оповещения о чрезвычайных ситуациях. В статье [6] решается задача применения позиционирования с помощью маркирования аудиосигналов для поиска видеопирата в зале кинотеатра. В статьях [7-9] разрабатывается новый метод маркирования аудиосигналов, а в статье [10] предлагается адаптация традиционного метода позиционирования с помощью этого метода маркирования для случая, когда взаимные расстояния между микрофоном видеопирата и акустическими динамиками, используемыми в помещении, определены с достаточной степенью точности, чтобы существовало вещественное решение системы из трех уравнений гипербол.

Однако, на практике не всегда удаётся обеспечить требуемую точность, что сказывается на эффективности позиционирования с учетом ограничений на размеры помещения. В этой статье предлагается комплексный метод позиционирования, основанный, как на традиционном подходе, основанном на точном решении системы из уравнений гипербол, так и на минимизации целевой функции, получаемой в результате применения метода наименьших квадратов при поиске приближенного решения такой системы уравнений.

\section{Формы прелставления шифрового ауАиосигнала}

Нормированный цифровой моно аудиосигнал можно представить в форме последовательности отсчетов - вещественных чисел, обычно принимающих значения из диапазона от -1 до 1:

$$
\mathbf{a}=a_{1} a_{2} a_{3} \ldots
$$

где $a_{i} \in[-1 ; 1]$, при этом $i \in\{1,2,3, \ldots\}$. Когда цифровой аудиосигнал является стерео аудиосигналом или имеет ещё больше, чем две аудиодорожки (например, квадро аудиосигнал), то такой сигнал можно представить в виде матрицы, каждая строка которой определяет отдельную аудиодорожку:

$$
\mathbf{A}=\left(\begin{array}{ccc}
A_{1,1} & A_{1,2} & \cdots \\
A_{2,1} & A_{2,2} & \cdots \\
\vdots & \vdots & \cdots \\
A_{N_{\mathrm{TX}}, 1} & A_{N_{\mathrm{TX}}, 2} & \cdots
\end{array}\right)
$$

где $N_{\mathrm{Tx}}-$ количество аудиодорожек; $A_{i, j} \in[-1 ; 1]$, при этом $i \in\left\{1,2, \ldots, N_{\mathrm{Tx}}\right\}, j \in\{1,2, \ldots\}$.

Для корректного вещания аудиосигнала, состоящего из нескольких аудиодорожек, требуется использовать несколько акустических излучателей (например, акустических динамиков) по количеству аудиодорожек.

\section{Комплексный метоА \\ позичионирования}

Для верного определения координат мобильного узла в трехмерном пространстве требуется как минимум четыре опорных узла. Таким образом, когда в качестве опорных узлов используются акустические излучатели, а в качестве мобильного узла - мобильное устройство с микрофоном, то для позиционирования в трехмерном пространстве требуется использовать четыре акустических динамика.

Пусть отправителем используются четыре динамика для вещания аудиосигналов с четырьмя аудиодорожками (то-есть $N_{\text {Тх }}=4$ ). Предположим, что отправитель с помощью метода маркирования, разрабатываемого в статьях [7-9], синхронно внедряет четыре маркера, каждый в отдельную аудиодорожку маркируемого цифрового аудиосигнала. Синхронное внедрение предполагает, что на стороне отправителя номера начальных отсчетов, с которых начинается внедрение каждого из маркеров, для всех четырех маркеров - одинаковые, то-есть передача маркеров во времени начинается одновременно со всех четырёх акустических динамиков.

Получатель, выполнив с помощью микрофона, например, стереозапись распространяющегося от акустических динамиков аудиосигнала, а затем, преобразовав записанный сигнал в цифровую форму, формирует таким образом цифровой стерео аудиосигнал:

$$
\mathbf{B}=\left(\begin{array}{lll}
B_{1,1} & B_{1,2} & \ldots \\
B_{2,1} & B_{2,2} & \ldots
\end{array}\right),
$$

где $B_{i, j} \in[-1 ; 1]$ - это отсчеты записанного цифрового аудиосигнала. Записанный микрофоном цифровой 
аудиосигнал можно рассматривать как комбинацию из аудиосигналов, переданных четырьмя акустическими динамиками отправителя. Предполагается, что частота выборки при преобразовании отправителем маркированного цифрового аудиосигнала в аналоговую форму равна частоте дискретизации при преобразовании получателем записанного с помощью микрофона аналогового аудиосигнала в цифровую форму.

В традиционных системах позиционирования мобильный узел обычно является активным участником процесса позиционирования, что обычно выражается в наличии протокола синхронизации между опорными узлами и мобильным узлом. Это, в свою очередь, позволяет выполнить оценку расстояний между мобильным узлом и опорными узлами. Далее эти оценки расстояний, а также известные координаты опорных узлов можно использовать для формирования и решения системы из уравнений сфер, в центре которых располагаются опорные узлы. В статье [11] можно найти сравнение численных методов приближенного решения таких систем. Получаемые таким образом точки пересечения сфер можно рассматривать как оценки координат мобильного узла.

Однако, когда позиционирование выполняется на основании записи аудиосигнала, начавшейся в произвольный момент времени, тогда для определения координат микрофона вместо оценки расстояний до динамиков можно использовать разницы в расстояниях между микрофоном и парами динамиков. Пусть получатель, применив предложенный в статьях $[9,12]$ метод обнаружения маркеров к записанному аудиосигналу в цифровой форме, определил номера отсчетов, с которых начинаются маркеры, отправленные посредством соответствующих акустических динамиков. Обозначим номера этих отсчетов символами: $n_{1, k}, n_{2, k}, n_{3, k}, n_{4, k}$ , при этом первый нижний индекс указывает на номер акустического динамика отправителя, а второй на номер маркера, отправленного соответствующим динамиком. Так как маркеры отправлялись во времени синхронно, то можно оценить разницу в расстояниях между микрофоном и парой динамиков, один из которых первый динамик, а другой - оставшийся из трех, по следующей формуле:

$$
R_{1, i, k}=\frac{n_{1, k}-n_{i, k}}{F_{s}} v,
$$

где $i \in\{2,3,4\}, F_{\mathrm{s}}$ - это частота дискретизации аналогового сигнала, записанного микрофоном получателя, $v$ - скорость распространения акустических волн в воздушной среде от акустических излучателей отправителя до микрофона получателя.

Пусть координаты $(x, y, z)$ акустических динамиков отправителя следующие: первый динамик $(0,0,0)$, вто- рой динамик $\left(x_{2}, 0,0\right)$, третий динамик $\left(x_{3}, y_{3}, 0\right)$, четвертый динамик $\left(x_{4}, y_{4}, z_{4}\right)$. Если координаты на практике окажутся не такими, то их всегда можно свести к таким с помощью смещения начала координат, вращений координатных осей и координатных плоскостей. Определение координат микрофона $\left(x_{\mathrm{M}}, y_{\mathrm{M}}, z_{\mathrm{M}}\right)$ получателя маркированного аудиосигнала осуществляется с помощью решения следующей системы из трех уравнений гипербол:

$$
\left\{\begin{array}{c}
\sqrt{x_{\mathrm{M}}^{2}+y_{\mathrm{M}}^{2}+z_{\mathrm{M}}^{2}}-\sqrt{\left(x_{\mathrm{M}}-x_{2}\right)^{2}+y_{\mathrm{M}}^{2}+z_{\mathrm{M}}^{2}}=R_{1,2, k}, \\
\sqrt{x_{\mathrm{M}}^{2}+y_{\mathrm{M}}^{2}+z_{\mathrm{M}}^{2}}-\sqrt{\left(x_{\mathrm{M}}-x_{3}\right)^{2}+\left(y_{\mathrm{M}}-y_{3}\right)^{2}+z_{\mathrm{M}}^{2}}=R_{1,3, k}, \\
\sqrt{x_{\mathrm{M}}^{2}+y_{\mathrm{M}}^{2}+z_{\mathrm{M}}^{2}}-\sqrt{\left(x_{\mathrm{M}}-x_{4}\right)^{2}+\left(y_{\mathrm{M}}-y_{4}\right)^{2}+\left(z_{\mathrm{M}}-z_{4}\right)^{2}}=R_{1,4, k} .
\end{array}\right.
$$

В статье [10] предложен метод решения такой системы. Когда эта система не имеет вещественного решения, тогда поиск координат осуществляется по методу наименьших квадратов, применённому к этой системе уравнений, что, по сути, заключается в поиске следующего минимума:

$$
\min _{\left(x_{\mathrm{M}}, y_{\mathrm{M}}, z_{\mathrm{M}}\right)} \mathrm{F}\left(x_{\mathrm{M}}, y_{\mathrm{M}}, z_{\mathrm{M}}, k\right) \text {. }
$$

где $\mathrm{F}\left(x_{\mathrm{M}}, y_{\mathrm{M}}, z_{\mathrm{M}}, k\right)$ обозначает следующую целевую функцию:

$$
\begin{aligned}
\mathrm{F}\left(x_{\mathrm{M}}, y_{\mathrm{M}}, z_{\mathrm{M}},\right. & k) \\
& =\left(\sqrt{x_{\mathrm{M}}^{2}+y_{\mathrm{M}}^{2}+z_{\mathrm{M}}^{2}}-\sqrt{\left(x_{\mathrm{M}}-x_{2}\right)^{2}+y_{\mathrm{M}}^{2}+z_{\mathrm{M}}^{2}}-R_{1,2, k}\right)^{2} \\
& +\left(\sqrt{x_{\mathrm{M}}^{2}+y_{\mathrm{M}}^{2}+z_{\mathrm{M}}^{2}}-\sqrt{\left(x_{\mathrm{M}}-x_{3}\right)^{2}+\left(y_{\mathrm{M}}-y_{3}\right)^{2}+z_{\mathrm{M}}^{2}}-R_{1,3, k}\right)^{2} \\
& +\left(\sqrt{x_{\mathrm{M}}^{2}+y_{\mathrm{M}}^{2}+z_{\mathrm{M}}^{2}}-\sqrt{\left(x_{\mathrm{M}}-x_{4}\right)^{2}+\left(y_{\mathrm{M}}-y_{4}\right)^{2}+\left(z_{\mathrm{M}}-z_{4}\right)^{2}}\right. \\
& \left.-R_{1,4, k}\right)^{2} .
\end{aligned}
$$

Так как требуется выполнять поиск координат $\left(x_{\mathrm{M}}, y_{\mathrm{M}}, z_{\mathrm{M}}\right)$ микрофона в ограниченном пространстве помещения, то при вынесении решения о возможном местоположении микрофона следует учитывать размеры помещения. Исчерпывающий поиск минимума $\mathrm{F}\left(x_{\mathrm{M}}, y_{\mathrm{M}}, z_{\mathrm{M}}, k\right)$, даже в ограниченном диапазоне значений аргументов функции, является вычислительно трудоёмкой задачей. Поэтому, обычно, для поиска минимума используются итеративные численные методы поиска, например алгоритм Давидона (Davidon), детально описанный в статье [11].

\section{Результаты натурных экспериментов}

Для оценки точности позиционирования с помощью маркированных аудиосигналов, полученных с помощью предложенного в статьях [7-9, 12] метода маркирования цифровых аудиосигналов, была собрана лабораторная установка и проведены натурные эксперименты по позиционированию в помещении. Лабораторная установка состояла из: 
- четырех акустических динамиком Edifier R1280DB;

- четырёх капсюльного микрофона AKG Lyra, настроенного на режим работы Tight Stereo (фронтальная стереозапись);

- компьютера, оснащённого звуковой картой Realtek ALC887, на базе материнской платы ASUS P8Z77-V LX;

- среды разработки MATLAB R2020а, использованной для создания программной составляющей модели аудиостегосистемы с множественным входом (несколько акустических динамиков) и множественным выходом (несколько микрофонов или многокапсюльный микрофон).

Для передачи маркированного аудиосигнала, состоящего из четырех аудиодорожек, использовались четыре динамика Edifier R1280DB $\left(N_{\mathrm{Tx}}=4\right)$, а для записи - микрофон AKG Lyra, настроенный на режим фронтальной стереозаписи.

Микрофон располагался В координатах $(x, y, z)=(0 ; 0 ; 203(\mathrm{~cm}))$. Нумерация динамиков, их координаты, а также нумерация аудиодорожек маркированного аудиосигнала, которые вещались ими, были следующими:

1-й динамик располагался в координатах $(0 ; 0 ; 0)$;

- 2-й динамик располагался в координатах (-23 см; $0 ; 0)$;

- 3-й динамик располагался в координатах (34 см; $10 \mathrm{~cm} ; 0)$;

- 4-й динамик располагался в координатах (17 см; $17 \mathrm{~cm} ;-30.5 \mathrm{~cm})$.

С помощью каждого из динамиков было отправлено по 10 маркеров, т.е. в общем было отправлено 40 маркеров; поэтому переменная $k$, представленная в вышеприведенных формулах, принимала значения от 1 до 10. Частота выборки маркированного цифрового аудиосигнала при вещании акустическими динамиками, также как и частота дискретизации в микрофоне обе были равными 44100 Гц. Параметры маркирования были такими, что маркер во времени занимал 44.41 секунды. Передача 40 маркеров (каждый динамик, одновременно с остальными, вещал соответствующую аудиодорожку, содержащую по 10 маркеров) с учетом заградительных интервалов (10\% от длительности маркера) между ними занимала

\section{$10 \cdot 1.1 \cdot 44.41$ сек $\cong 488.51$ сек.}

С помощью микрофона, работающего в режиме стерео, была получена запись, состоящая из двух аудиодорожек. Каждая из двух записанных им аудиодорожек рассматривалась как комбинация аудиодорожек, которые вещались каждым из четырех акустических динамиков. Аудиодорожки, представляя собой векторы, подвергались анализу для решения задачи позиционирования. На основании записанных двух аудиодорожек были получены 30 замеров разниц в расстояниях между микрофоном и парами динамиков: по 10 замеров для каждой из двух записанных аудиодорожек и 10 замеров для суммы аудиодорожек (переход в моно аудиосигнал).

Поиск минимума функции $\mathrm{F}\left(x_{\mathrm{M}}, y_{\mathrm{M}}, z_{\mathrm{M}}, k\right)$ выполнялся с помощью средств математической программы MATLAB с учетом следующих значений границ помещения: $\left|x_{\mathrm{M}}\right|<220 \mathrm{~cm},\left|y_{\mathrm{M}}\right|<220 \mathrm{~cm},\left|z_{\mathrm{M}}\right|<220 \mathrm{~cm}$. Из 30 полученных замеров 8 замеров привели к точкам минимума с координатами, выходящими за границы помещения. Точки минимума, расположенные за границами помещения не учитывались при оценке статистических показателей качества разрабатываемого комплексного метода позиционирования. Итак, натурные эксперименты показали следующее:

- среднее значение расстояний между найденными координатами микрофона и истинными координатами оказалось равным 145 (см)

- стандартное отклонение расстояний между найденными координатами микрофона и истинными координатами оказалось равным 31 (см).

В статье [6] утверждается, что с помощью метода маркирования Nakashima-Tachibana-Babaguchi (использованы фамилии авторов указанной статьи) достигается среднее значение расстояний между вычисленными и истинными координатами микрофона равное 40 (см), при этом стандартное отклонение составляет 133 (см). Однако, метод маркирования, предложенный в статье [6], в отличие от разработанного в статьях [7-9, 12] метода маркирования, для достижения указанных показателей требует таких значений сил встраивания маркера, при которых становятся значительно слышимыми акустические артефакты от результатов внедрения маркера. Таким образом, разработанный в статьях [7-9, 12] метод маркирования цифровых аудиосигналов, позволяет достичь хороших показателей точности позиционирования при такой силе встраивания маркеров, которая обеспечивает отсутствие слышимости артефактов от внедрения маркера в аудиосигнал.

\section{Зак^ючение}

В статье был предложен комплексный метод позиционирования маркированными аудиосигналами в помещении. Выполнена статистическая оценка точности позиционирования с помощью предложенного 
комплексного метода. В качестве статистических показателей были использованы стандартное отклонение и среднее значение расстояний между координатной точкой истинного месторасположения микрофона и координатными точками, найденными в результате минимизации целевой функции по данным, полученным из натурных экспериментов по передаче и при- ёму маркированных аудиосигналов. Статистические показатели точности позиционирования, полученные по результатам натурных экспериментов, показывают принципиальную возможность позиционирования в помещении маркированными аудиосигналами, полученными с помощью методов маркирования, предложенными в статьях [7-9, 12].

\section{ЛИТЕРАТУРА}

1. Dardari D., Closas P., Djurić P.M. Indoor tracking: Theory, methods, and technologies // IEEE Transactions on Vehicular Technology.— 2015. — T. 64. — № . 4.-C. 1263-1278.

2. Liu H., Darabi H., Banerjee P., Liu J. Survey of wireless indoor positioning techniques and systems // IEEE Transactions on Systems, Man, and Cybernetics, Part C (Applications and Reviews).— 2007.— T. 37.— №.6.—C. 1067-1080.

3. Alarifi A., Al-Salman A., Alsaleh M., Alnafessah A., Al-Hadhrami S., Al-Ammar M.A., Al-Khalifa H.S. Ultra wideband indoor positioning technologies: Analysis and recent advances // Sensors. — 2016. — T. 16. — № . 5.— https://doi.org/10.3390/s16050707

4. Mesmoudi A., Feham M., Labraoui N. Wireless sensor networks localization algorithms: a comprehensive survey // arXiv preprint arXiv:1312.4082. — 2013.

5. Moreno D., Ochoa S.F. Understanding the resource positioning methods that support mobile collaboration // 2016 IEEE International Conference on Systems, Man, and Cybernetics (SMC). - 2016. D0I:10.1109/SMC.2016.7844805.

6. Nakashima Y., Tachibana R., Babaguchi N. Watermarked movie soundtrack finds the position of the camcorder in a theater // IEEE Transactions on Multimedia. 2009. - T. 11. - № . 3.- C. 443-454.

7. Гофман М.В. Методика скрытой передачи данных при связи через воздушный аудиоканал // Труды СПИИРАН. 2017. Вып 2. С. $97-22$.

8. Гофман М.В., Корниенко А.А., Мирончиков Е.Т., Никитин А.Б. Цифровое маркирование аудиосигналов для робастной скрытой акустической связи через воздушный аудиоканал // Труды СПИИРАН. - 2017.- Вып. 6. - С. 185-215.

9. Гофман М.В., Корниенко А.А., Глухарев М.Л. Метод детектирования маркера в цифровом аудиосигнале авторизованным получателем // Проблемы информационной безопасности. Компьютерные системы. - 2020. - Вып. 4. - С. 58-71

10. Гофман М.В. Корниенко А.А., Глухов А.П. Методика позиционирования маркированными аудиосигналами // Проблемы информационной безопасности. Компьютерные системы. - 2018. — № 4. - С. 120-129.

11. Kanaan M., Pahlavan K. A comparison of wireless geolocation algorithms in the indoor environment //2004 IEEE wireless communications and networking conference (IEEE Cat. No. 04TH8733). — IEEE, 2004. — T. 1.—C. 177-182.

12. Гофман М.В. Разработка модели многоканальной аудиостегосистемы на основе маркирования цифровых аудиосигналов // Современная наука: актуальные проблемы теории и практики. Серия «Естественные и технические науки»_—2021 г.—№ 6. стр. 78-83.—D0I 10.37882/2223-2966.2021.06.11 